\title{
Memantine plus donepezil improves physical and mental health in people with Alzheimer's disease
}

Tariot PN, Farlow MR, Grossberg GT, et al. Memantine treatment in patients with moderate to severe Alzheimer disease already receiving donepezil. A randomized controlled trial. JAMA 2004;291:317-24.

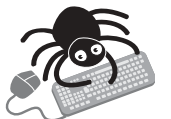

This article

contains extra text on the $\mathrm{EBMH}$ website

Does memantine in combination with donepezil improve physical and mental health of people with moderate to severe Alzheimer's disease?

METHODS

$\square$

Design: Randomised placebo controlled trial.

Allocation: Concealed.

Blinding: Double blinded.

Follow up period: Twenty four weeks.

Setting: Thirty seven sites, USA; June 2001 to June 2002.

Patients: 404 people receiving donepezil treatment for moderate to severe Alzheimer's disease (minimum age 50 years;

5-10 mg/day donepezil; MMSE score 5-14), living in the community with a caregiver willing to participate in the study. Exclusions: B12 or folate deficiency, ischaemia, or other major physical or mental disease.

D Intervention: Memantine (starting dose $5 \mathrm{mg} /$ day, increased by $\mathbf{R}_{\mathbf{x}}$ $5 \mathrm{mg} /$ week to final dose of $20 \mathrm{mg} /$ day); placebo.

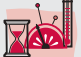

Outcomes: Primary outcomes: cognitive dysfunction (Severe Impairment Battery (SIB)); activities of daily living (modified 19 item Alzheimer's disease Co-operative Study-Activities of Daily Living Inventory (ADCS-ADL 19$)$ ). Secondary outcomes: effects of medication (Clinician's Interview-Based Impression of Change Plus Caregiver Input (CIBIC-Plus)); frequency and severity of behavioural symptoms (Neuropsychiatric Inventory (NPI)), and cognition, functioning and behaviour (Behavioral Rating Scale for Geriatric Patients (BGP)).

Patient follow up: Memantine: 172/203 (85\%); placebo: 150/ 201 (75\%).

\section{MAIN RESULTS}

At 24 weeks, memantine significantly improved cognition (SIB, $\mathrm{p}<0.001$ ) and function (ADCS-ADL $19, \mathrm{p}=0.03$ ), compared with placebo (see http://www.ebmentalhealth.com/supplemental for table). Memantine improved overall clinical response (CIBC-Plus, $\mathrm{p}=0.03$ ) and reduced behavioural disturbances and psychiatric

For correspondence: Pierre N Tariot, Departments of Psychiatry, Medicine Neurology, and the Center for Aging and Developmental Biology, University of Rochester Medical Center, Monroe Community Hospital, Rochester, New York, NY, USA; pierre_tariot@urmc.rochester.edu

Sources of funding: the Forest Research Institute, a division of Forest Laboratories Inc. symptoms (NPI, p=0.002), compared with placebo. Increased need for care was also significantly improved (BGP, $p=0.001)$. Memantine was generally safe and well tolerated (adverse events: $78 \%$ memantine $v 72 \%$ placebo), however confusion and headache occurred in at least 5\% of the memantine group (confusion: $7.9 \%$ memantine $v 2.0 \%$ placebo, $\mathrm{p}=0.01$; headache: $6.4 \%$ memantine $v$ $2.5 \%$ placebo, $\mathrm{p}=0.09)$. More people receiving placebo discontinued the trial ( $12.4 \%$ placebo $v 7.4 \%$ memantine).

\section{CONCLUSIONS}

Memantine significantly improves physical and mental health in people with severe to moderate Alzheimer's taking donepezil.

\section{Commentary}

M any patients with Alzheimer's disease (AD) take cholinesterase inhibitors (ChEls), but early studies of memantine necessarily excluded such patients. This important study is the largest RCT of memantine and is the first to assess combination with cholinesterase inhibitors (ChEls).

Important questions remain. Does memantine slow the rate of disease progression or, like the ChEls, give a symptomatic boost? (The randomised follow up was too short to reveal whether the memantine and placebo groups were diverging.) Does memantine affect institutionalisation and/or mortality? Both are questions with significant pharmacoeconomic implications but inevitably not addressed in licensing studies. What are predictors of treatment response?

The key question is whether the beneficial effect seen in clinical trials is apparent in practice. The usual biases might have enhanced the effect size but two effects might have reduced it: the lower dropout rate in the memantine group and the "noise" of patients at varying points in their response to donepezil. Furthermore, the clinical global measure is significantly different between drug and placebo. Arguably this means that, by definition, there is a clinically significant effect.

So, would I prescribe memantine? Yes, in moderate and severe AD. Small changes in ADLs, care dependency, and behavioural disturbances in this patient group are more likely to have important repercussions on care needs than, for example, in mild disease. Experience with ChEls suggests that even when changes in other measures are small, the clinical global measure should not be ignored.

Memantine does not seem to cause the early improvement in ADLs often seen on initiating ChEls. This makes it difficult to make decisions about prolonged and continued treatment based on an initial assessment of treatment response. However, this strategy, although mandated for ChEls by NICE in the UK, never had any evidence to support it: treatment response at 3 months does not predict reponse at 6 months. Once again, it looks as if stopping the drug will be more difficult than starting it. Perhaps we should stop worrying about this and start to accept that, as with other chronic diseases, long term treatment will be the norm in $A D$ too.

Rupert H McShane, MD, MRCPsych Honorary Senior Clinical Lecturer and Consultant in Old Age Psychiatry, University of Oxford and Oxfordshire Mental Healthcare Trust, Oxford, 